\title{
Hikikomori, a Phenomenon of Social Withdrawal and Isolation in Young Adults Marked by an Anomic Response to Coping Difficulties: A Qualitative Study Exploring Individual Experiences from First- and Second-Person Perspectives
}

\author{
Roseline Kim Fong Yong1,2, Yoshihiro Kaneko' \\ ${ }^{1}$ Department of Public Health, Graduate School of Medicine, Akita University, Akita, Japan \\ ${ }^{2}$ Department of Mental Health, Graduate School of Medicine, The University of Tokyo, Tokyo, Japan \\ Email: yangjinhong@gmail.com
}

Received 11 November 2015; accepted 12 January 2016; published 15 January 2016

Copyright (C) 2016 by authors and Scientific Research Publishing Inc.

This work is licensed under the Creative Commons Attribution International License (CC BY). http://creativecommons.org/licenses/by/4.0/

(c) (i) Open Access

\section{Abstract}

Hikikomori is a recent phenomenon among young adults in which they isolate themselves from social contacts and responsibility for more than 6 months. This problem has assumed epidemic proportions in Japan and has been widely discussed across disciplines. Previous studies have associated hikikomori with a maladaptation to structural change, an emergent mental problem that needs new diagnosis, or an under-diagnosed schizophrenic disorder. Most previous work has adopted a clinical perspective, and there have been no qualitative studies exploring the individual feelings of people suffering from the syndrome. We adopted a qualitative method using a grounded theory approach. Data collection and analysis took place between September 2006 and August 2008. Theoretical sampling included eight informants from snowball sampling and an online observation consisting of 160 online participants. Analysis focused on informants' first- and secondperson experiences of hikikomori. Data analysis revealed one overriding theme: coping difficulties consisting of the two categories stasis and expression. As a result of conflicting demands and reduced autonomy, respondents experienced stasis, which prevented them from moving forward; "hiding" or "avoiding" heightened the expression of their behaviors. This is the first study to explore the in-depth experience of individuals suffering from hikikomori syndrome in a non-clinical setting. We argue that hikikomoriis not a result of asocial behavior, but rather an anomic response to a situation that informants felt powerless to change and from which they could see no way out. 
We also observed that feelings of hopelessness and relationship fatigue might be overcome by introducing a relaxed social environment that people can control during rehabilitation.

\title{
Keywords
}

\author{
Hikikomori, Social Withdraw, Social Isolation, Individual Experiences, Qualitative Study, Coping \\ Difficulties, Mental Health
}

\section{Introduction}

Hikikomori was first defined by Japanese researchers as a unique social withdrawal and isolation syndrome that describes young adults not engaged in work or study, not in the process of finding a job, and who have had no relationships with others (except family members) for more than 6 months; additionally, behavior in hikikomori is not related to psychiatric problems [1]. Hikikomori syndrome can arise from any cause. The word has recently made its way into the Oxford Dictionary of English and features in international social media, where it is the topic of numerous video clips, films, anime, and articles (Appendix). As media depictions of hikikomori have always been associated, rather dramatically, with suicide and crime, the word has a negative connotation, resulting in extra emotional stress to sufferers and their family members. Parents of children with hikikomori often report insufficient social support [2]. Hikikomori syndrome has had a tremendous social impact. The phenomenon attracted international academic attention as early as 2002 with a review article in The Lancet suggesting that hikikomori was a new and urgent public health problem [3]. A 2011 article in the same journal suggested that hikikomoriwas an undiagnosed mental illness that was rapidly spreading to countries outside of Japan [4].

One research project [5] has estimated that 25,000 (0.72\%) teenagers and young adults (aged 15 - 34 ) in Tokyo suffer from hikikomori. A national survey in 2010 reported 696,000 people with hikikomori (including those who only leave the house for specific reasons) [6]. It has also been reported that $1.2 \%$ of the Japanese population have hadhikikomori experiences and that $0.5 \%$ of households have at least one child with hikikomori [7]. Speculation that hikikomori is a culturallybound phenomenon [8] [9] has been disproved, as similar syndromes continue to emerge overseas, with cases in Oman [10], Spain [11], South Korea [12], and Canada [13]. A telephone survey suggested that $1.9 \%$ of young adults in Hong Kong between 12 and 29 years fit the international label of hikikomori, which specifies social withdrawal of more than 6 months, and 2.5\% have experienced social withdrawal for less than 6 months [14].

Research in Japan indicates that half of people who have suffered from hikikomori syndrome in the past have no experience of mental illness, while the half that have suffered from mental illness have only experienced mood disorders [7]. This differentiates hikikomori from common social withdrawal, as the latter is always a symptom of a pre-existing mental illness, such as schizophrenia. However, the new national consensus is that although there is no empirical evidence that hikikomori is equivalent to common social withdrawal, schizophrenia may be under diagnosed in people with hikikomori [15] Clinical guidelines advise that hikikomori's association with developmental disorder and other common mental illnesses (e.g., social phobia and adjustment disorder) should not be ignored [15]. Reasoning that hikikomori may stem from social phobia or pervasive developmental disorders, Kondo et al. [16] believe that hikikomori patients can be diagnosed according to criteria in the Diagnostic and Statistical Manual of Mental Disorders, Fourth Edition, Text revision [17]. Kato et al. [18] conducted a survey among clinicians in other parts of Asia, and in Australia and the United States. Using an example of a patient presenting with hikikomori syndrome, they asked the clinicians if they had seen similar cases, and if they had, what diagnosis they would give to the patient. All the participating clinicians recognized such a case from their own clinical settings and indicated that they would choose a diagnosis of a common mental illness.

The Japan Ministry of Health, Labor and Welfare has declared that hikikomori syndrome can have several causes. Consequently, the phenomenon has drawn the attention of specialists from different fields, including sociologists, psychiatrists, and psychologists, which has resulted in different interpretations of hikikomori. There is a continuing debate about whether hikikomori is a symptom of existing mental illness or a newly developed disease. There has also been discussion about whether the syndrome is just a social phenomenon that has noth- 
ing to do with psychological disability. The opinions of international clinicians differ regarding the nature of hikikomori. A range of clinical diagnoses are given to people who develop hikikomori, and there has been speculation about the involvement of social environment, family relationships, personality, mood, cultural factors, and about the presence of psychosis [18]. The role of pressure to conform in hikikomorihas also been highlighted [10] [19]-[21]. The etiology of hikikomori is still under speculation, and a number of theories have been suggested: that the syndrome is an anomic response of individuals in relation to changing opportunity structures [20]; a common outcome of a multiplicity of social, medical, or emotional problems when individual needs are neglected to emphasize conformity; a social sickness [19] [22]; or post-traumatic stress disorder as a result of parenting style [9]. Researchers have suggested that hikikomori youths have narcissistic beliefs [23], that they manifest the laziness of spoiled youth [24], or that hikikomori is perhaps a personal choice [25] [26]. A self-reported case study suggested that an introverted personality plays a crucial role in a person becoming a hikikomori and that hikikomori develops from a combination of environmental and personal factors [13]. Hikikomori has been associated with maternal panic disorder and parents with a high level of education, but not with parenting styles [27].

These inconsistent findings are probably a result of focusing too much on theory rather than on sufferers' actual experiences. This reflects the poor consensus in attitudes to medical and psychiatric treatment of the disorder [28]. Interpretation of hikikomori has mainly been based on samples obtained from medical institutions, rehabilitation centers, or youth support centers. This may explain why the research findings to date are biased toward mental health diagnoses. However, sociological research on hikikomori is also limited, as it has focused mainly on observations rather than on dialogue. Socially withdrawn youths seldom seek help [7], and of those that do, not many remain in treatment or continue to access the services provided [28], suggesting that currently available services do not meet their needs. This may explain why people with hikikomori resist treatment and refuse to be labeled [19]. It is possible that the longer people experience hikikomori, the better quality of life they have [26], which means that support and help may be unnecessary. However, most studies suggest that people with hikikomori may suffer from undiagnosed mental health issues [16] [19], indicating that they do need support and treatment. People are most likely to seek clinical treatment once they feel they are ready to, or following the intervention of troubled parents. As hikikomori results from the interplay between psychological, social, and behavioral factors [29], treatment can be extremely difficult, especially if it is based on a narrow specialist perspective.

Although much has been written about hikikomori syndrome, current studies lack the voice of the individuals who experience social withdrawal and have no interpersonal relationships with people-other than family members-for more than 6 months. Are these individuals happy with their situation? What do they think about their situation? We believe that to provide more effective cross-perspective treatments,it is vital to identify what hikikomori means from the individual's perspective. However, it is extremely difficult to gather data from the individual before their situation gets wild. In most cases, when they were presented to the clinical settings, they had developed comorbidities such as having delusions, behaving violently, or attempting suicide. Could the progress of developing comorbidities in mental illness be avoided? We considered that hints may lieinthe individual perspectives of the hikikomori sufferers. Previous work had studied people in clinical and rehabilitation settings [9] [10] [16] [23] [28] [30]-[33], multiplayer online gamers [26], students [34], individuals who have recovered from hikikomori in community surveys [7] [27], experts [18] [35], and individuals participating in telephone surveys [14]. We wondered whether it was possible to approach people with hikikomori syndrome who had not attended clinical institutions or rehabilitation centers and did not engage in multiplayer online gaming. In addition, we believed that there are insufficient personal descriptions of the phenomenon, and that most theories about hikikomori are based on quantitative studies that depend on expert opinion rather than individual experience. We therefore set out to obtain a more comprehensive phenomenological description of the hikikomori experience using a qualitative method with non-clinical samples and by setting up an investigating team with mixed expertise. Using snowball sampling, our aim was to explore the question: "What is it like to be in a social withdrawal state for more than 6 months without maintaining interpersonal relationships with others?”

\section{Methods}

\subsection{Research Team and Reflexivity}

The investigation team consisted of two investigators with mixed expertise. The primary investigator ( $\mathrm{PhD}$, 
MPH) is a multi-linguist who speaks English, Chinese, Malay, and Japanese, and is familiar with American, Chinese, and Southeast Asian culture. At the time of the study, this researcher was a freelance translator for several private and government institutions. She was trained in pharmaceuticals, public health, and mental health, and has listening and counseling skills. Her work requires her to deal with prison inmates, deaf people, immigrants, and asylum seekers who feel socially isolated. This researcher carried out the interviews in this study. The second investigator ( $\mathrm{PhD}, \mathrm{MD}$ ) is an academic who specializes in suicide prevention, is fluent in English and Japanese, is familiar with Japanese culture, and is well trained in clinical pharmacology and public health. Both investigators had no previous established relationships with the informants prior to the study commencement.

An assumption was made prior to the study commencement that the existing studies and reports do not represent a first-person perspective of hikikomori and are based more on expert opinion. Therefore, we wanted to give voice to the silent sufferers of hikikomori, to compare and contrast how respondents' views of reality conflicted with current knowledge. The primary investigator was particularly interested in why a person would isolate themselves for more than 6 months for no particular reason. The second investigator was particularly interested in informants' well-being. Informants knew that the aim of the investigation was to better understand the hikikomori syndrome from a first-person perspective.

\subsection{Study Design}

We wanted to envisage the basic social processes between events that the informants experienced. For this reason, we used unstructured qualitative interviews and a constructive grounded theory approach [36] to explore the issues in depth. There were no presets of questions, but the questions were built around the $5 \mathrm{~W} 1 \mathrm{H}$ principle (why, where, who, when, what, how), which was informed by the participants. Informants were recruited through snowball sampling. To maximize the richness of the data, multiple routes were explored for data collection, which took place between September 2006 and December 2008.

\subsubsection{Sample Inclusion and Exclusion Criteria}

As our aim was to explore individual experiences from first- or second-person perspectives, we limited our theoretical sampling to persons (or family members of persons) that had experienced social withdrawal and isolation for more than 6 months without associating with others and without a clinical diagnosis of mental illness or mental retardation. Persons with physical disabilities and mental illness were excluded from the study.

\subsubsection{Theoretical Sampling Process}

Simultaneous with snowball sampling, we extended our data collection to documentaries, creative productions, and casual conversations with random people to ascertain what they thought about the phenomenon. Sample recruitment was conducted through personal networks, international conferences, seminars, university cafes, and university student halls by posting recruitment information and distribution of a hikikomori awareness bookmark with a contact email printed on it. To gain a wider perspective on hikikomorisyndrome, we advertised for participants in English, Chinese, and Japanese. Announcements were made at several international conferences held in Hong Kong and Korea. Participants of these conferences were mainly from China, Korea, Japan, India,the United States, Australia, New Zealand, and parts of Europe. Sample recruitment in mental health clinics and medical facilities was carefully avoided to exclude expert influence.

The open invitation approach generated 58 potential participants during the ground sampling, and two (Informants A and B) were recruited via theoretical sampling. Informant A was recruited through a seminar in Hong Kong. She had a cousin 2 years older than herself who had intermittently confined herself in her room for 8 years. Several interview sessions of 2 - 3 hours were conducted in informal settings, cafes, and a conversation corner in a library. Informant B was recruited at an international conference in Korea. This participant had a sister 2 years younger than himself who had confined herself to her room for about 2 years. Two interviews were conducted in the hotel lobby; each lasted for an hour.

All interviews were digitally recorded, transcribed in detail by the primary investigator, and the transcripts checked against the recording by the secondary investigator. Interview transcripts were analyzed and case-based memos were written during and after each interview. The data, memo writing, and coding were compared from sample to sample to refine the theoretical sampling. 
With the information gained from the first two informants, we began our second sampling recruitment through the Internet. A Google keyword search of the term "hikikomori" was performed and emails were sent to a selection of individuals who were responsible for blogs, and who had identified themselves as suffering from hikikomori, inviting them to take part in interviews. Of the five bloggers we approached, one responded to our interview request, Informant C. He had experienced hikikomori for 8 years and had been in recovery from the syndrome for 4 years at the time of interview. Online email interviews were conducted with this participant. The emails were rather short and contained a few open-ended questions based on his description of the hikikomori experience. We found that although he agreed to the interview, it was not pleasant for him to describe his hikikomori experience. Informant $\mathrm{C}$ was more interested in describing his present beliefs and exploring whether he could help other people with similar experiences. At the end of the session, he sent us his diary for further analysis.

Based on the data collected, we sent emails and chat invitations to 78 potential informants with screen names that contained the following terms: "hikikomori", "loneliness", "unwanted", "shadow", and "failure" using Internet social media such as MSN Messenger, Skype, and Mixi (a Japanese social networking service popular in the 1990s). Twenty-five individuals responded to our interview request. After a preliminary interview, 21 people were excluded as they had either chosen their screen name for amusement and did not have hikikomori syndrome or they had experienced hikikomori for less than 6 months. Both Informants D and E were recruited through Skype and both had the screen name "hikikomori". Informant D was a Japanese man, in the process of recovering from 8 years of hikikomori syndrome. We conducted an interview with him through a Skype call that lasted for only 10 minutes and we engaged in several Skype chats with him. He was not a frequent online user. Informant $\mathrm{E}$ was an Austrian man who had suffered from hikikomori for about 2 years. Interviews were conducted through 12 Skype chats that lasted 2 - 8 hours per chat. The interviews were his first attempts to chat online with others; he had previously only used his account for online file transfer.

After comparing and contrasting the data, codes, and memos, we set up a hikikomori interest group on Facebook with a brief definition of hikikomori to extend our theoretical sampling. Five members joined the group, one of whom, Informant F, was eligible for theoretical sampling. He had a younger sister who had isolated herself in her room for almost 10 years since the age of 10. She spoke only to her mother and refused to leave the house. The interviews were conducted over four MSN chats and two separate emails in which this informant told us about his sister and gave us his interpretation of her problem.

Based on theoretical sampling, we approached Informant $\mathrm{G}$ whom we identified through an online channel designed for Japanese-speaking people in Japan with hikikomori. Informant $C$ had told us about this site. Informant $\mathrm{G}$ was the site manager and had suffered from hikikomori for 4 years. The site is run by people with hikikomori for others with the syndrome; it hosts several bulletin boards and chat rooms that allow chatters to sign in with a nickname and to read the chats without participating. There was no description of the source of funding for the site and no evidence of specialist intervention was found in the posts.

At this stage, we had almost achieved data saturation; however, to complement our findings from the snowball sampling, we used online observation to compare and contrast participants' experiences while developing the theoretical themes. We observed several chat rooms and bulletin boards on the site managed by Informant $\mathrm{G}$. We matched the computer IDs to the screen names to make sure that there were no duplicate or ghost users. We had not obtained any single personal information from the IP address. In other words, the computer ID did not allow us to identify the person or compromise the privacy of the person. We assumed that when more than two computer IDs shared the same screen name, the participant may have logged in at an Internet cafe or from computers in a public venue; in this case, the data was excluded from the analysis as it did not fulfil the inclusion criteria. Using these criteria, 160 participants were observed with the informed consent of Informant G. Through the online observations, we managed to recruit one of the most active chatters, Informant $\mathrm{H}$, as our last theoretical sampling informant. She had experienced hikikomori for 20 years and spent a large amount of time chatting online when she was at home. The flow of the theoretical sampling and the data collected are shown in Figure 1.

\subsubsection{Interview Guide}

To build a comprehensive description of hikikomori, we began by posing the question: What characterizes the experience of hikikomori? Open-ended questions such as "How long have you/he/she experienced hikikomori?", "What do you/he/she normally do?”, “How do you feel?”, or "What do you think she/he feels?” were directed to informants in a casual way to encourage conversation flow. Simple prompts were often used to facilitate the in- 


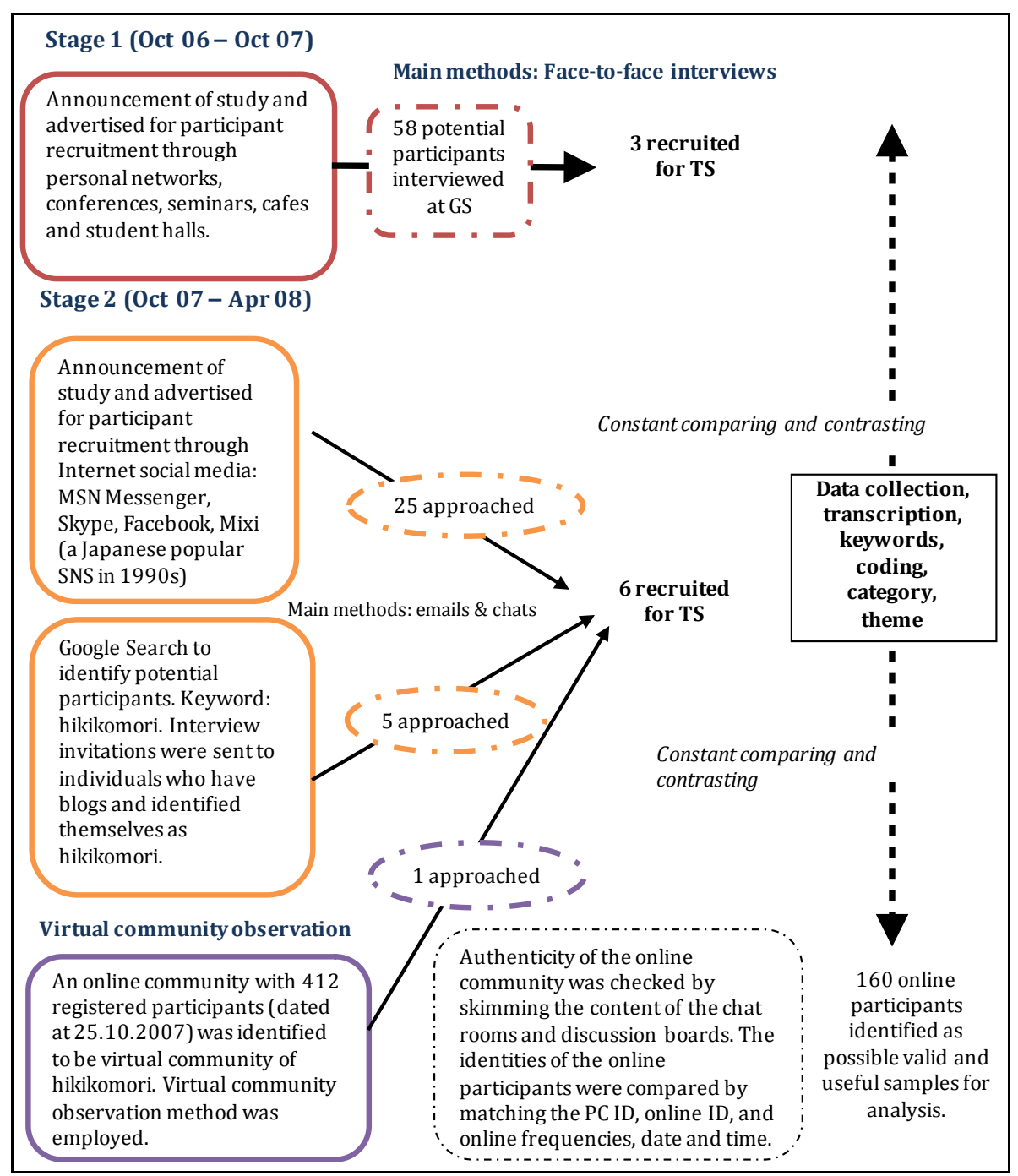

Figure 1. Participant recruitment flow from grounded sampling (GS) to theoretical sampling (TS).

terview process. The informants were encouraged to talk spontaneously and to take as much time as they needed to respond. The interview depth and duration depended on the response, susceptibility, and openness of each informant, who could stop the interview at any time. This protected the informant and minimized undue pressure, which is particularly important as hikikomori are characterized by social interaction avoidance. The investigator stopped the interview dialogue when sufficient information was gathered or when the interviewee refused to proceed. Interviews were conducted using various methods: telephone, face-to-face, email, and Internet chat whenever the interviewee was willing. The interview method was determined by what was feasible and appropriate for each respondent's circumstances. Informants controlled the flow of the conversation to facilitate their comfort and enable them to relax during the interview. Informants also decided the time and place of their interview sessions. Face-to-face and telephone interviews were audio recorded and later transcribed verbatim by the primary investigator. Interviews were generally carried out over a few sessions. In each subsequent interview, the investigator reviewed what had been discussed in the previous interview, to allow informants to reflect and elaborate on their experiences.

\subsubsection{Online Observation Guide}

This method made it impossible to verify any of the participants' identities. Multiple observations over time were made. Chat-room activity and discussion-board posts were recorded in detail and compared and contrasted 
with the data obtained from one-to-one interviews. In each observation, data collection gave focus and priority to the discussion topics, the in-depth responses of the participants, and the chat room behavior of the participants. Focus was particularly given to the time lag in responding to a message, the habit of leaving and rejoining the group, relationship with chatters, and the frequency with which participants spent more than 48 hours online on working days.

\subsubsection{Description of Data Analysis}

After each interview, the primary investigator listened to the recordings and reorganized the observational notes prior to transcription. Transcripts and printed dialogues were recorded in their original language. Data analysis was conducted simultaneously with data collection. The primary investigator wrote notes and memos while reading the transcripts and developed tentative ideas about categories and relationships according to the patterns observed in each informant's interviews. To illustrate this paper, examples of text from the interviews were translated into English. All investigators compared preliminary coding results before doing any further coding, and ambiguities were iteratively revisited until consensus was reached. Figure 2 shows an example of how initial coding was developed from an interview and how they contributed to the categorization of the codes before being incorporated into the themes.

Chat-room and discussion-board data were disassembled into constituent semantic units within each conversation and compared against the categories and ideas derived from interviews. Particular attention was paid to retaining contextual information. Data collection and analysis was conducted by constant comparison among the samples and with the literature to develop a theoretical sampling framework. As theoretical themes emerged, we reviewed the codes again and redefined them. The process of theoretical sampling and data analysis was repeated until the data were saturated. We read and reread the content of all data, removing redundancy, extracting commonalities and features, and hierarchically organizing the data into themes, categories, and elements. Selected representative quotes are used here to exemplify the themes and categories. Original verbatim quotes in English were used without altering the grammar or sentence structure, whereas verbatim quotes in Japanese or Chinese were translated as precisely as possible to preserve their original meaning. This study was reported in accordance to the consolidated criteria for reporting qualitative research (COREQ) checklist [37].

\subsubsection{Ethics and Confidentiality}

For the direct interviews, individual informants were informed of the study purpose and of their right to confidentiality and uncontested withdrawal. In all cases of snowball sampling, written and oral informed consent was obtained from all individual informants included in the study. With the consent of the informants, the interviews were audio taped (if conducted face-to-face or over the telephone) to facilitate verbatim transcription and subsequent data analysis. For interviews conducted through Internet chat or emails, after obtaining individual consent a detailed transcript of the conversation was recorded, printed, and retained in an easily retrievable format. These observations were compared and contrasted with the one-to-one interviews to break down the codes and define the categories.

Informed consent for the online observations was sought from the site manager, Informant G. The online community was set up so that personal information was not traceable with computer ID, all participation was

\begin{tabular}{|l|l|}
\hline Line-to-line coding & Interview statements \\
\hline $\begin{array}{l}\text { Suspicion } \\
\text { Antipathy }\end{array}$ & "I don't trust them ...the distrust and dislike because of \\
my experience...I condemn most people that I see or & have to talk to, because of my misanthro- \\
Trauma from the past & pist-thinking...As a result I condemned most of them...I \\
wisapproval of others & betrayed by somebody you first have to trust someone." \\
Asocial behavior & \\
Justifying & $\begin{array}{l}\text { Memo writing: } \\
\text { During the interview, the case continued to play games while chatting. Answers were always prompt, unless the conversation caught } \\
\text { his attention. In responding to the question, "Whydon't you make friends?" he paused and thought for a few minutes and typed his } \\
\text { reply, "I don't trust them......" What caught my attention was that, he had referred "friends" as "them", which suggested that } \\
\text { "friends" were there in his mind. Yet the unpleasant experience (he was heavily bullied in school while he was in his teens) in hispast } \\
\text { created trauma and resulted in a strong mistrust towards others. Yes, mistrust, that is the word. He was so suspicious to others that he } \\
\text { preferred to spend time alone. }\end{array}$ \\
\hline
\end{tabular}

Figure 2. Example of the line-by-line coding of an interview statement and memo writing. 
anonymous or identified by a logged in screen name, the participants' identities were not recoverable. We monitored the computer IDs to enable us to restrict sample inclusion to one screen name per computer ID, which enhanced the trustworthiness of the sample. However, this did not reveal personal information that would allow us to track each individual. Informed consent from the site manager was considered to be sufficient: as these observations took place via the Internet, they were de facto published, and hence were in the public domain. The messages in their original form are not retrievable using the direct quotes as a query in search engines, and all responses were anonymous. All verbatim quotes were translated into English for the purpose of this study. Ethical approval was obtained from the Institutional Review Board of the University of Hong Kong.

\section{Results}

\subsection{Informants' Characteristics}

Data were obtained from eight one-to-one interviews and online observation of 160 individual site participants. Seven of the one-to-one interview informants were recruited using snowball sampling, and one was recruited through online observation. Of the seven individuals recruited by snowball sampling, three were proxy respondents who had a family member with hikikomori and four had experienced hikikomori themselves for 1 year or more (Table 1). Each informant therefore described a case of a person suffering from hikikomori syndrome. Three informants were women and five were men. Five cases had become hikikomori during their teens, one in her mid-30s, and one at 11 years. Three cases were not Japanese and lived outside Japan. Interviews with the hikikomori cases were often short and showed signs of disconnection in the conversations. The situation often improved after several interview sessions.

Chat-room participants were demographically indistinct, unless they voluntarily disclosed their details on line. Gender was often identifiable through gender-specific language use, but other demographic information was unavailable. Computer ID rather than username was used to identify and track contributions from different individuals. Using these criteria, 160 participants were observed. Among them, 30 were men, 9 were women, and the remaining 121 participants were of unknown gender.

Data analysis revealed one overriding theme, that of coping difficulties. Hikikomori was seen by most respondents as a reaction to difficulties faced in daily living, people, job demands, personal demands, disability, disappointment, unpleasant situations, and fear and mistrust, among other things. Two categories comprised this theme of coping difficulties: stasis and expression. As a result of conflicting demands and reduced autonomy, respondents experienced stasis, which prevented them from moving forward; as they failed to move forward, "hiding" or "avoiding" heightened the expression of their behaviors. The informants felt that they had stopped moving forward, and going online expressed their attempts to maintain the stasis situation.

\subsection{Core Category 1: Stasis}

This first category described the characteristics of hikikomori and encapsulated a range of trigger events and

Table 1. Demographics of the informants recruited using snowball sampling.

\begin{tabular}{|c|c|c|c|c|c|c|c|c|c|c|}
\hline \multicolumn{3}{|c|}{ Informant } & \multicolumn{7}{|c|}{ Hikikomori case } & \multirow{2}{*}{$\begin{array}{c}\text { Interview } \\
\text { sessions }\end{array}$} \\
\hline & Age & Gender & Relationship & Age & Gender & Ethnicity & Status & Location & Year & \\
\hline A & 24 & Woman & Cousin & 27 & Woman & Chinese & Current & China & 8 & Face-to-face/3x \\
\hline B & 38 & Man & Sister & 35 & Woman & Korean & Current & Korea & 2 & Face-to-face/2× \\
\hline $\mathrm{C}$ & 33 & Man & Self & 33 & Man & Japanese & Previous & Japan & 8 & Email/6× \\
\hline $\mathrm{D}$ & 28 & Man & Self & 28 & Man & Japanese & Current & Japan & 8 & $\begin{array}{l}\text { Skype chat/2× } \\
\text { Phone call/1× }\end{array}$ \\
\hline $\mathrm{E}$ & 24 & Man & Self & 24 & Man & Caucasian & Current & Austria & 2 & Skype chat/12× \\
\hline $\mathrm{F}$ & 24 & Man & Sister & 21 & Woman & Japanese & Current & Japan & 19 & $\begin{array}{c}\text { MSN chat } / 4 \times \\
\text { Email/2× }\end{array}$ \\
\hline G & 24 & Man & Self & 24 & Man & Japanese & Current & Japan & 4 & Email/3× \\
\hline $\mathrm{H}$ & 38 & Woman & Self & 38 & Woman & Japanese & Current & Japan & 20 & Skype chat/12× \\
\hline
\end{tabular}


reactions: experienced weakness, non-competitiveness, ineffective communication skills, failure, stress, pride, job insecurity, slander, and bullying, which prompted respondents to move to a psychological space where they could exist unchallenged. These features were concatenated into four elements that comprised the category of stasis: "hopelessness", "relationship fatigue”, "inevitability”, and "fear".

\subsubsection{Hopelessness}

A number of features characterized this element, including perception of a harsh world, feelings of being helpless in the world, and disappointment in work life. Although informants had experienced social withdrawal for more than 6 months, most were still traumatized by their experiences before their withdrawal from society. In their descriptions of the past, they often remarked that the world was simply too harsh to cope with. This illustrated a felt sense of incompetence, a feeling that they were unable to deliver what the world expected from them. Life was an ill-matched fight in which they were unable to contend.

The first time I worked, the world was so harsh, and taking responsibility at work was too hard for me. I had to quit. I picked up a free employment magazine, went home, looked through it; I became depressed, and I just couldn't break out from it.

To some, total withdrawal marks resignation from the stiff competition they faced in the real world. Some emphasized a sense of hopelessness about the future and felt helpless about not having a secure job. Hikikomori appeared as a response to social defeat.

[I became hikikomori]... when I was 25 years old. I had started [to] refuse school since [my] school days. At my first working place, [I] resigned after one year. And after that, my jobs were only for a few months, back and forth in between NEET. Now, I am over 30, totally defeated, a hikikomori. I guess that is my position.

NEET is anacronym that describes young people who are "Not in Education, Employment, or Training”. The word was first coined in the United Kingdom. In Japan, NEET is often compared with hikikomori [20] [38]. Globalization and other economic trends have generated significant numbers of social casualties for whom secure life-long employment has been replaced by contractual appointments, generating a sense of loss of occupational and social security as well as job prospects. Hikikomori has been described as the inevitable consequence of a social system that has collapsed, suggesting that the phenomenon will become increasingly prevalent as more people are incorporated into this system of employment [20] [38].

$[L]$ ost job, being retrenched, the company closed down... being retrenched, the company closed down... [difficult breathing, gasping]... [back to normal] disappointment in love, no friends, constantly in fear, sick...

Some respondents felt that they had been overloaded. Too much was expected from them, and because of these unreasonable demands, their "operating systems" had "crashed".

It's like being a computer coping with tasks that are beyond its capability; it continued doing that every day untilit collapsed.

It was like too many unfortunate things piled together on me. I just want to start all over again.

\subsubsection{Relationship Fatigue}

This element encapsulated social difficulties comprising aspects of identity, difficult interactions, and low selfesteem. One informant described his sister who was slowly recovering from hikikomori. Hiding away in her room was her way of coping with identity issues arising from unpleasant relationships at home and in the workplace.

My sister worked for a non-profit organization making video animations before she became a hikikomori. She saw many people fighting each other in the company, and she received many negative comments. At home, she also had a lot of negative feedback from our stepmother and elder sister-in-law since she was young... She had one very intimate friend, but she cut off the relationship with her too. She recently told me that her intimate friend was working together with her in the same company before she resigned. I guess she felt betrayed.

Sometimes, the decision to withdraw seemed to be the best solution to respondents' perceived limited social 
abilities.

It was difficult [for me] to socialize with people.

For some, loneliness seemed to be a less painful alternative than coping with other people.

If it is such a pain to think about whom you should have fun with, it may be better to find a way to have fun alone.

\subsubsection{Inevitability}

The lack of alternatives perceived by respondents and a sense of inevitability in their fates led to a withdrawal into hikikomori. This belief of inevitability seemed to play a central role in the decision to socially withdraw, rather than any perceived "inability." Many felt caught in a situation because they had no other choice. For example, one chat-room participant demonstrated no difficulties during online communication and was able to lead the participants in several discussion topics, but felt unable to organize a simple sentence when facing real people, which impaired conversation.

In fact, it's not only about [the fact that] I can't complete a conversation, but even if I could possibly laugh over it, and respond by nodding or saying "yeah" to people, but...

There were often long pauses in the conversations before the informants began to express themselves, yet when they started talking, we could sense their struggle, their difficulty in completing the ideas or stories they initiated. Sometimes, perceived social invisibility contributed to low self-esteem, undermining confidence.

[L]acking recognition by others, I couldn't cope... The fact was, I had no self-confidence.

Another metaphor that was used to describe respondents' lack of competitiveness in the job market was the idea of being faulty, viewing themselves as defective commodities, resulting in a sense of deficiency.

I couldn't get a job, although I was a graduate. I was just like defective goods.

...[T] he free magazine [Author note: this free magazine is distributed at train stations and contains job advertisements] or the Sunday news advertisement, I took them home, but then after I looked through them, I thought I just couldn't deliver what they want. I just can't do it...

Respondents perceived themselves as unfit even though they were often overqualified for jobs. This perhaps reflects a reaction to a failure of social recognition linked intimately to occupational worth.

\subsubsection{Fear}

One informant was confused about his sister's behavior. She suggested that her sister had dropped out of school because of peer comments about her appearance. Her sister had refused to attend school and hid herself from all her relatives, avoiding seeing or talking to anyone else other than her immediate household family members. She never stepped out of the house. Her room became her own territory, where she felt secure.

I think her friends had gossiped about her behind her back, and it hurt her... she also seems to have a fear of making eye contact with others, and she is afraid of comments about her face. I think she's trying very hard just to make conversation with us at home. She can never make eye contact and stuff. Somehow, I felt that it must be her friends that had made her feel that way.

Some respondents felt that their weakness meant that their only option was to hide away from society.

I am a weak person. I can only hide in the dark; I am that kind of person.

Having “dropped out", people who experienced hikikomori often felt rejected because they were different.

Society says what is good and what is not and most people go with the flow... so if you can't go with the flow, you are the evil one... [Investigator: "But I thought you said you won't call yourself a bad person?] Sure I'm not, but in the eyes of society I am, because I think too differently to be able to go with the others.

Withdrawal was also used to cope with disappointed expectations. By avoiding others, a person would not be reminded of their disappointments or challenged by them, and lived in a world in which their beliefs would not 
be challenged.

She was not satisfied with her bachelor's major... she didn't want others to observe her negative status... she always thought of herself as the smartest and prettiest girl in the world, much better than we cousins, thus she only wants to work in the best company and get the best position-but is that possible? She never faces the truth... we are always talking about our studies, work, boyfriends, and even apartments and cars, but she has none.

The elements of statis highlighted the challenges of coping with the daily demands of life in a world where many feel alienated and anomic; they embody a feeling of mismatch rather than simple disaffection. Hikikomori sufferers often view the external world as a battlefield. For many, the sense of being defeated by the world was strong. This suggests that hikikomori arises as a result of being unable or unwilling to cope with and compete in social engagements. Instead of expressing adaptive proactive coping to overcome conflicts, or self-adjustment to better match the situation, a more emotion-focused strategy of withdrawal was adopted. Thus, hikikomori could be a strategy to reject change, and perhaps a mark of resignation from the past and present harshness of contemporary life: the actualization of alienation.

\subsection{Core Category 2: Expression}

The core category expression emerged from the online observations and described how social withdrawal extended into participation in online community. Lack of expression is a vicious circle that embodies the elements that help maintain the hikikomori syndrome: "mistrust", "hindrance", "anonymity", and "transposition".

\subsubsection{Mistrust}

Trust was often destroyed by disappointment or past negative experiences, which constructed a self-defense mechanism, resulting in asocial behavior. One informant explained that his experience of being brutally bullied at school led to a general distrust of people that was damaging to subsequent relationships.

I don't trust them... the distrust and dislike because of my experience... I condemn most people that I see or have to talk to, because of my misanthropist thinking... As a result, I condemned most of them... I won't even trust them to hold a letter for me... To get betrayed by somebody you first have to trust someone...

By becoming hikikomori, respondents avoid interpersonal relationships and the uncertainty surrounding them.

A smart strategy to prevent myself getting hurt is not to have intimate relationship with others.

Respondents' conversations in the chat rooms and on the bulletin boards featured this key element of trust, which made sense as they discussed how they feared interactions. As we compared and contrasted interviewees' feelings about trust with their background stories, we began to see that Internet interaction was not something that they naturally preferred. However, it allowed them to avoid face-to-face interaction, which they disliked because of unpleasant experiences and unexpressed fear.

\subsubsection{Hindrance}

Most respondents feared human relationships to some degree. Some confessed being very cautious of establishing Internet relationships, taking a considerable time to observe the chat rooms and bulletin boards before contemplating joining. This underlying fear of relationships and inability to trust became a significant hindrance for them. Mistrust and fear merged to determine what they could and could not achieve.

Because I'm always caught in fear...

I didn't know what to trust... I was so suspicious that it was like I was being swallowed by my own suspicions, and I hate this... because of fear, I can't even enter the chat room. I'm able to write on the bulletin boards. Is there a site that hikikomori can trust?

To some, even playing player-versus-player (PVP) online games remained a huge challenge. For example, an informant told us that although he recognized that playing and sharing with others might be more satisfying, he continued playing alone.

I play online games a bit. When I beat the monster, I feel happy. Yet there would also be times when I would 
think, perhaps if I had played with someone else as a team, wouldn't it be more fun?

On the surface, the respondents may seem to be enjoying the freedom of being alone, yet the choice to remain alone probably stems from a fear of disapproval from others, which prevents people with hikikomori from socializing.

I enjoy playing alone [pauses]... yes [pauses]... I do. Why bother to play with others? Not everyone has similar thoughts to yourself; it is better to live alone.

Because they felt hindered in exploring new relationships, respondents had convinced themselves that they were happier being alone.

No one bothers about the others. It is just about the game. We enjoy the game, that's all, not the relationship.

Although experience hindered them from making social contacts with others, the desire to communicate with others led respondents to adopt a tactic of anonymity.

\subsubsection{Anonymity}

Respondents experienced doubt even in virtual environments where, despite participating in online social interactions, they preferred to remain anonymous. Some participated only when the chat room guaranteed total privacy.

Please, create a chat room that allows us to sign in without giving names.

On [name of site], you can chat without giving your name.

Some responses suggested that informants had never really opened up or invested trust in people around them. It was hard for them to find a social group in which they felt safe and comfortable enough to share their thoughts and feelings.

It is hard to find people [like myself], most likely because those who are like me rarely share their inner feelings or thoughts with others.

The preference for anonymity suggested that they wanted a channel through which they could freely express their thoughts and feelings without being identified. For some, Internet relationships were as far as they would go. Informant $\mathrm{H}$ was an active and outspoken participant in the hikikomori virtual community and on chat media, but baulked at face-to-face and even telephone meetings. Regarding the virtual friendship that she built over the Internet, she said:

It's tough for me to even have a telephone conversation with others... maybe there are really nice people in reality, but at the present, I am just not in the mood to meet any.

\subsubsection{Transposition}

Despite the fear and distrust of real world and face-to-face interactions, some respondents maintained a regular online presence and even a community. Unlike in the real world, this enabled them to share social support and interactions while retaining complete control over the context of the interactions. Possessing no identity means that negative social consequences such as loss of face can be avoided if someone withdraws abruptly from the chat room; these negative experiences are almost impossible to avoid in real life conversations. As the other users were also socially withdrawn, respondents felt much safer to express themselves in this online setting. Therefore, although many respondents failed to trust others and were fearful of social engagement, they did not completely cease their social interactions; rather, they transposed them from an uncontrollable and threatening reality to a more controllable virtual social world. Within this safer environment, people with hikikomori could rebuild their confidence in social interactions in a controlled and less threatening manner.

"Meeting" good people on the net would help to reduce the feeling of distrust toward people.

Virtual interactions reduce the information load from body language that can contradict verbal communication and create ambiguity and uncertainty in social interactions. However, virtual interactions can reduce the accuracy of communication. 
In real[ity], [you] get to see the facial expression and the body language of the people that [you] speak to, and you might read [perceive] some kind of message from that.

The virtual chat room represents real-time interactions. To participate, respondents had to log in to the chat room. Their screen name would then appear and the chat room moderator would identify the participant and inform others about his or her existence. Disembodied conversations between identifiable participants are thus possible. Intriguingly, while some respondents logged in as "anonymous", most consistently used identifiable screen names, presenting a clear, though unknown, identity. In this way, virtual friendships formed as users became familiar with each other.

Despite this, fear and hopelessness dominated the respondents and impaired trust on certain issues. These disappointing experiences generate negative self-evaluations, which hinder more positive adaptive responses and handicap interactions, even in virtual relationships. Nonetheless, these are tolerated and often overcome as respondents build up the confidence to interact from a safe place. Expression documents the externalization of feelings of mistrust and fear in people with hikikomori and this outward expression appears to be a coping strategy to enable them to overcome disappointment in the past, and fear of people and the future.

\section{Discussion}

The study focused on how the informants felt about their experience of hikikomori, either in themselves or members of their immediate family. The informants talked about their past experiences and described their concerns about their present situations. In contrast to Chan and Lo's findings [25], our respondents did not describe hikikomori as an active, pleasant choice, but as an alternative, passive way of coping with existing problems. The coping features of hikikomori are not culturally specific, but are more likely to be a specific adaptation to contemporary competitive social changes and the human relationships these changes produce. This suggests the possibility of a growing cross-cultural phenomenon, as some researchers have observed [18] [19] [28].

\subsection{The Hikikomori Process: A Push and Pull Effect}

While the category of stasis describes how powerless respondents felt, the category of expression reflects the way in which that insecurity undermined trust in human relationships. Stasis includes rejection experiences; coping difficulties at school, work, and with people; self-limiting attitudes about one's inability to cope with these difficulties; and a sense of inevitability about life in general, as opposed to actively coping by adjusting one's behavior, attitude, or situation. Previous studies had reported that social withdrawal can release individuals from the pressure to perform social responsibilities and from the need to be fast and efficient [27] [37]. In contrast, our respondents indicated that, whether they liked their situationor not, they felt stuck when they finally realized how bad their situation was. Hikikomori behavior inevitably becomes the only real solution, a static position of hiding away adopted to reject change demanded by disappointment and subsequent loss of visibility and mistrust of others - an alternative to adaptive coping with disappointment. Anomic coping and fear maintain withdrawal and subsequently hinder efforts to regain trust. Different manifestations of this process seem to perpetuate hikikomori, which appears to be an anomic response to life and interpersonal relationships. However, people with hikikomori do not always reject human relationships, as illustrated by the elements of "anonymity" and "transposition" that we identified.

We found that the practice of anonymity triggered transposition. Online interaction allows anonymity, which permits people to express themselves freely without fear of being judged or criticized by others. Instead, a protected, guarded, or controlled tentative interaction with others can occur via online meetings; as confidence builds, new identities are adopted and used. This process helps respondents to reorganize their thoughts. However, as the conversations are almost always virtual and anonymity, this transposes the experience of being "unwilling or unable to communicate" to that of being "unwilling or unable to leave the Internet." This coping behavior explains how online activities helped respondents to maintain the state of hikikomori and why people with hikikomori tend to also engage in compulsive Internet use [32]. People with hikikomori syndrome may rely heavily on the Internet to fill their natural need to communicate with others. However, engagement in an online community does not necessarily guarantee satisfying social relationships for socially withdrawn people.

Consistent with other reports [21], we found that communication difficulties sometimes led to avoidance of human relationships. This analysis suggests a more complex coping response featuring intact social needs un- 
derpinned by a deep distrust of relationships. Our findings suggest that the dominant view that people with hikikomori close off all social communication with the outside world and alienate themselves, withdrawing into their own space is imprecise. This is how the disorder appears superficially to family members. However, their extensive chat-room participation and engagement with bulletin boards suggest that many people with hikikomori are not ready to relinquish all forms of communication. Many hikikomori sufferers have created a comfort zone where things are more predictable and less challenging. Once established there, their inability to re-emerge could explain why people with hikikomori have difficulties re-engaging with social activities. Mistrust and fear emerge as determinants of what can and cannot be achieved. The experiences of our informants suggest that Internet chat sites might play an important interim step in the first attempts to retest the trustworthiness of others. We agree with previous observations that virtual social networks can help people with hikikomori to redefine their social identity to be more positive, as well as receive peer support and recognition through developing virtual social networks and intimacy [24] [25]. We argue that these people can accept a more controllable form of interaction. This could be seen as a latent function of the behavior of seeking social support; thus, the virtual community appears to be an important means of social engagement. However, this conclusion may simply reflect the sampling method chosen, and there are almost certainly people with hikikomori who are completely isolated. Nonetheless, controlled interaction environments may provide one means by which people with hikikomori could be encouraged to begin rebuilding confidence in relationships within a sheltered environment, and these environments might constitute an important therapeutic component.

Our findings indicate that people with hikikomori are unwilling or afraid to participate in social interaction and prefer the predictability of withdrawal to the uncertainty of social interaction. Although this may appear to be a choice the person has made, it is their feelings of hopelessness and relationship fatigue with others that have pushed them toward seeking withdrawal, and their sense of inevitability and fear that has pulled them into stasis. In addition, prolonged social disengagement weakens a person's social survival skills, making it even harder for them to re-engage. Respondents expressed a fear of leaving their comfort zone, despite their belief that they had no control over their situation. In this sense, hikikomori is similar to other anxiety-linked states-in both, anticipated negative consequences generate high levels of anticipatory anxiety to which the person has low tolerance and which inhibit them from testing the situation. Our data suggest that mistrust of others and distancing are strong contributors to the prolonging of social-withdrawal syndrome. Although a less threatening online community is introduced to replace the stressful environment in which hikikomori sufferers live, the disorder becomes an expression of the interactions among mistrust, hindrance, anonymity, and transposition that sustain their stasis.

\subsection{What Could Be Done to Help Hikikomori?}

\subsubsection{Ineffective Communication within Family}

We found that there is a discrepancy between the information provided by family members and that given by individuals who have experienced hikikomori themselves. In this study, family members tended to focus on the probable association of hikikomori with poor interpersonal relationships, whereas individual hikikomori stressed issues with self-esteem, self-performance and fear of the future. Family members felt that hikikomori is the preferred choice, whereas hikikomori felt that their condition is unavoidable. Although our data does not provide enough evidence to pinpoint what has gone wrong or what could have be done to prevent hikikomori, our observations highlight deficient communication between parents and children. Although the hikikomori and family members are living under the same roof, communication among family members was probably inadequate even before the person became a hikikomori. This study raises the question of whether hikikomori can be prevented if early symptoms are spotted.

\subsubsection{Surprising Communication Power}

In our study, we ensured that the time, place, and context of the interviews were as informal as possible. One advantage of this approach is that, while the participants may initially feel tense, they gradually become more open and are able to communicate their stories. The preliminary interviews were often very short and did not touch on the kind of information we were seeking, but subsequent interviews were more fruitful as participants became more comfortable expressing themselves. Informants often showed signs of disconnection in the first and second interviews, but then slowly began to open up. Some interviews lasted for hours. Interactions among 
the virtual community users were similar to those in the one-to-one interviews. This suggests that people with hikikomori may not be as asocial as previously assumed. Rather, if they are given the time to organize their thoughts during a conversation, they are able to engage and are generally happy to communicate their thoughts.

\subsubsection{Communication Can Be Therapeutic}

We realized that informants were able to communicate with others when provided with an environment over which they had control. In fact, one of our informants resumed work and began new relationship with others 4 months after being interviewed. In a follow-up study, he revealed that apparently the unstructured interviews had allowed him to review what happened to him in the past. In doing so, he discovered what he really wanted and decided to move on. Previously, he had never spoken about himself to others. The dialogue helped him to reflect on himself and this helped him to decide what to do next. Although there was no intended intervention during the interviews, the opportunity to communicate could have facilitated this change.

\subsubsection{Suggestions to Current Approaches: Installing Self-Motivation to Change}

Existing approaches to treating hikikomori include medical treatment [14], social-skills training [27] and providing counseling and a place to belong [22]. These are essentially top-down approaches that effectively say "there is a problem here, let's fix it; to fix it, you've got to do it this way." The characteristics of our informants and what we observed through the Internet forum caused us to question the efficacy of these approaches. First, a huge portion of hikikomori do not seek medical treatment and probably never will. Second, many of them are grown adults, who have acquired active learning skills. While people in hikikomori situations may seem passive, our observations reveal that they can be proactive when provided with the right conditions. We suggest that future approaches should include talking sessions that allow hikikomori to express themselves freely with the goal of facilitating self-reflection. Self-reflection may provide self-motivation to change. To achieve that, we encouraged the informants to express their feelings in their own words, as this makes it easier to evaluate where the problem lies and how it is connected with what they really want. Once they are able to visualize this, they can initiate the next step.

\subsection{Originality and Richness of the Data}

Unlike previous studies, this study attempted to sample people who have severe or have experienced severe hikikomori syndrome outside of a clinical setting. This is also the first international study that has considered the perspectives of actual hikikomori rather than depending on specialists' opinions. This study sought to understand the hikikomori experience from the perspective of people with the syndrome. The trustworthiness of the analysis was supported throughout by two multilingual investigators and further strengthened by the fact that the primary investigator conducted all the interviews. The interviews varied in richness and included a wide range of experiences. Leading questions were carefully avoided; rather, informants' expressions were repeated back to them to encourage them to ponder on their thoughts and to share further. The non-directed interview style encouraged the informants to talk freely about their experiences without any restrictions, thus enhancing the credibility of the findings. All the informants claimed that this was the first time they had told their stories to others.

All the informants in this study, both those in Japan and those in other countries, called themselves hikikomori, suggesting that there is a widespread self-awareness of the problem. In the preliminary screening process, we found that some informants did not feel negatively about being hikikomori sufferers, whereas others felt ashamed. Of the eight informants from the theoretical sampling, those who were family members of hikikomori sufferers felt that hikikomori was an avoidance behavior. In contrast, informants who had experienced hikikomori syndrome themselves viewed it as an unavoidable action that they had taken; none were proud of their situation. All of these respondents had had hikikomori syndrome for more than 2 years; none were diagnosed as psychotic, and none maintained long-term online relationships with others.

Although we obtained a non-clinical hikikomori sample and used open, non-directional questions, all the face-to-face interviews were long and resulted in rich but subtle information as well as non-verbal information. Interpreting non-verbal information is challenging, as there is a danger of the interviewer's assumptions biasing their interpretation. We thus decided to include the online observations as supplementary data in this study. Online observations were carefully documented and analyzed to compare and contrast them with the interviews. We also compared the online bulletin board behaviors with behavior from other common social network system 
bulletin boards. We found that the common social network system bulletin boards often featured rapid replies from members and contained substantial critical conversations. In contrast, members of the hikikomori online bulletin boards were slow to reply, sometimes taking days to respond to a posted message. Conversations on the hikikomori boards were often more empathic than critical. These features of this online community suggest that its members had hikikomori or social withdrawal and that they were being very cautious with their words.

\subsection{Study Limitations and Future Directions}

This study examined a sample of individuals who were socially withdrawn at the time of the study, individuals in transition or recovering from hikikomori, and individuals who had emerged from a period of hikikomori, to produce rich descriptions of people's experiences of this poorly understood condition. As the aim of a qualitative study is to build rather than test theories, we are aware that our samples are not fully representative of all people with hikikomori and that our hypothesis cannot be generalized to the whole population. Also, as our participants were not clinically assessed and were self-diagnosed as suffering from "hikikomori", it is unclear how consistent and clinically relevant the sample is. We deliberately sampled non-clinical participants as our ultimate goal was to gain insights into the experiences of this group of people. Although the characteristics of the online community members that we observed suggested that they had hikikomori, we could not verify these respondents' hikikomori diagnoses. However, the online users had declared themselves to be suffering from hikikomori, and we included only those participants who had spent a substantial amount of time on the chat and discussion sessions during the daytime on working days. Although we analyzed data from both Japanese and nonJapanese informants, the hypothesis that we constructed could be biased to Japanese samples as the online community is located in Japan, and hosted by Japanese people. The interpretations of the data may also be subject to bias from the investigators' assumptions.

In our study, we theoretically sampled people that presented with hikikomori syndrome and the family members of people with hikikomori. We suggest that future research should focus solely on firsthand experiences of hikikomori syndrome, rather than including family members of people with hikikomori. Future questions might include the following: Are people with hikikomori unhappy with their situation? Do they wish to recover from it? Had they tried seeking help from others before they became socially withdrawn? Had they tried seeking assistance from others while they were socially withdrawn? Is it possible to provide early interventions before people develop hikikomori syndrome? Although quantitative studies may be useful in investigating commonalities of the disorder, more qualitative studies are needed to understand the personal experiences of hikikomori.

\section{Conclusion}

This is the first study to explore in-depth experiences of people currently suffering from, or with past experiences of, hikikomori syndrome. This is also the first attempt to analyze non-clinical data. We argue that hikikomori syndrome is not the result of asocial tendencies, but rather an anomic response to a situation that people feel powerless to change and from which they can see no way out. Feelings of hopelessness and relationship fatigue may be overcome by introducing a relaxed social environment that people can control during rehabilitation from hikikomori. Exposing sufferers to new, interesting ideas and allowing them to try (and fail) may widen their perspectives and help them to overcome fear. Treatment remedies should focus on regaining trust and monitoring the anxiety symptoms that prevent them from trying new things.

\section{Acknowledgements}

We thank Richard Fielding for inspiring this study and mentoring the data-collecting process as well as his input on initial data analysis. We also thank Josh Onishi and Hannah Mitchell in assisting for the preliminary analysis of scripts. We thank Yuki Miyamoto for her contribution to the secondary analysis of the data and many others who have helped to facilitate the interviews and follow-up studies. We also like to thank Simon Pleasants for editing the final draft.

\section{References}

[1] Saito T. (1998) Shakaiteki hikikomori: Owaranai shishunki. (Societal hikikomori: Unending adolescency). PHP-Kenkyujo, Tokyo. 
[2] Funakoshi, A. and Miyamoto, Y. (2014) Significant Factors in Family Difficulties for Fathers and Mothers Who Use Support Services for Children with Hikikomori. Psychiatry and Clinical Neuroscience, 69, 210-9.

http://dx.doi.org/10.1111/pcn.12230

[3] Watts, J. (2002) Public Health Experts Concerned about “Hikikomori”. Lancet, 359, 1131.

http://linkinghub.elsevier.com/retrieve/pii/S0140673602081862 http://dx.doi.org/10.1016/S0140-6736(02)08186-2

[4] Kato, T.A., Shinfuku, N., Sartorius, N. and Kanba, S. (2011) Are Japan’s Hikikomori and Depression in Young People Spreading Abroad? Lancet, 378, 1070.

http://www.thelancet.com/journals/lancet/article/PIIS0140-6736(11)61475-X/abstract http://dx.doi.org/10.1016/S0140-6736(11)61475-X

[5] Takatsuka, Y. (2008) Jittai chousa kara miru hikikomori wakamono kokoro (a Glimpse to the Young People’s Mind through Field Research on Actual Condition). In: Tokyo Metropolitan Government Office for Youth Affairs and Public Safety, Ed.

[6] Director General for Policy on Cohesive Society (2010) Wakamono no ishiki ni kansuru chousa (hikikomori ni kansuru jittai chousa) (An Attitude Survey of Young People: The Actual Situation of Hikikomori). In: Cabinet Office of Policy on Cohesive Society, Ed.

[7] Koyama, A., Miyake, Y., Kawakami, N., Tsuchiya, M., Tachimori, H., Takeshima, T., et al. (2010) Lifetime Prevalence, Psychiatric Comorbidity and Demographic Correlates of "Hikikomori” in a Community Population in Japan. Psychiatry Research, 176, 69-74. http://linkinghub.elsevier.com/retrieve/pii/S0165-1781(08)00387-9 http://dx.doi.org/10.1016/j.psychres.2008.10.019

[8] Teo, A.R. (2010) A New Form of Social Withdrawal in Japan: A Review of Hikikomori. International Journal of Social Psychiatry, 56, 178-85. http://isp.sagepub.com/cgi/pmidlookup?view=long\&pmid=19567455 http://dx.doi.org/10.1177/0020764008100629

[9] Hattori, Y. (2006) Social Withdrawal in Japanese Youth: A Case Study of Thirty-Five Hikikomori Clients. Journal of Trauma Practice, 4, 22. http://www.tandfonline.com/doi/abs/10.1300/J189v04n03_01 http://dx.doi.org/10.1300/J189v04n03_01

[10] Sakamoto, N., Martin, R.G., Kumano, H., Kuboki, T. and Al-Adawi, S. (2005) Hikikomori, Is It a Culture-Reactive or Culture-Bound Syndrome? Nidotherapy and a Clinical Vignette from Oman. International Journal of Psychiatry in Medicine, 35, 191-198. http://ijp.sagepub.com/cgi/pmidlookup?view=long\&pmid=16240975 http://dx.doi.org/10.2190/7WEQ-216D-TVNH-PQJ1

[11] Garcia-Campayo, J., Alda, M., Sobradiel, N. and Sanz Abos, B. (2007) A Case Report of Hikikomori in Spain. Medicina Clinica, 129, 318-319.

[12] Lee, K., Koo, J., Kim, E. and Lee, S. (2001) The Psychosocial Characteristics of Oiettolie Adolescents. Korean J Counsel \&Pyschotherapy, 13, 147-162. (In Korean)

[13] Chong, S. and Chan, K. (2012) A Case Study of a Chinese "Hikikomorian” in Canada-Theorizing the Process of Hikikomorization. Journal of Special Education and Rehabilitation, 13, 99-114. http://dl.fzf.ukim.edu.mk/index.php/jser/article/view/840/838

[14] Wong, P.W., Li, T.M., Chan, M., Law, Y., Chau, M., Cheng, C., et al. (2014) The Prevalence and Correlates of Severe Social Withdrawal (Hikikomori) in Hong Kong: A Cross-Sectional Telephone-Based Survey Study. International Journal of Social Psychiatry, 61, 330-342. http://isp.sagepub.com/cgi/pmidlookup?view=long\&pmid=25063752 http://dx.doi.org/10.1177/0020764014543711

[15] Saito, K. (2010) Hikikomori no hyouka: Shien ni kansuru gaidorain (Evaluation and Support Guideline for Hikikomori). Ministry of Health, Labour and Welfare, Tokyo.

[16] Kondo, N., Sakai, M., Kuroda, Y., Kiyota, Y., Kitabata, Y. and Kurosawa, M. (2013) General Condition of Hikikomori (Prolonged Social Withdrawal) in Japan: Psychiatric Diagnosis and Outcome in Mental Health Welfare Centers. International Journal of Social Psychiatry, 59, 79-86. http://dx.doi.org/10.1177/0020764011423611 http://isp.sagepub.com/content/early/2011/11/16/0020764011423611.full.pdf+html?ijkey=Yd2F4d1rn3j0w\&keytype=r ef\&siteid=spisp

[17] American Psychiatric Association (2000) Diagnostic and Statistical Manual of Mental Disorders. 4th Edition, Text Revision (DSM-IV-TR), American Psychiatric Association, Arlington, 943 p.

[18] Kato, T., Tateno, M., Shinfuku, N., Fujisawa, D., Teo, A.R., Sartorius, N., et al. (2012) Does the "Hikikomor" Syndrome of Social Withdrawal Exist outside Japan? A Preliminary International Investigation. Social Psychiatry and Psychiatric Epidemiology, 47, 1061-1075. http://dx.doi.org/10.1007/s00127-011-0411-7

[19] Borovoy, A. (2008) Japan’s Hidden Youths: Mainstreaming the Emotionally Distressed in Japan. Culture, Medicine and Psychiatry, 32, 552-576. http://link.springer.com/article/10.1007\%2Fs11013-008-9106-2 
http://dx.doi.org/10.1007/s11013-008-9106-2

[20] Furlong, A. (2008) The Japanese Hikikomori Phenomenon: Acute Social Withdrawal among Young People. Sociological Review, 56, 309-325. http://onlinelibrary.wiley.com/doi/10.1111/j.1467-954X.2008.00790.x/epdf http://dx.doi.org/10.1111/j.1467-954X.2008.00790.x

[21] Toivonen, T., Norasakkunkit, V. and Uchida, Y. (2011) Unable to Conform, Unwilling to Rebel? Youth, Culture, and Motivation in Globalizing Japan. Frontiers in Psychology, 2, 207. http://dx.doi.org/10.3389/fpsyg.2011.00207 http://www.ncbi.nlm.nih.gov/pmc/articles/PMC3171786/

[22] Tomita, F. (1994) Yomigaetta kazoku no kizuna. Hato Shuppan, Toyo, 400 p.

[23] Ogino, T. (2004) Managing Categorization and Social Withdrawal in Japan: Rehabilitation Process in a Private Support Group for Hikikomorians. International Journal of Japanese Sociology, 13, 120-133. http://onlinelibrary.wiley.com/doi/10.1111/j.1475-6781.2004.00057.x/epdf http://dx.doi.org/10.1111/j.1475-6781.2004.00057.x

[24] Tran, M. (2006) Unable or Unwilling to Leave the Nest? An Analysis and Evaluation of Japanese Parasite Single Theories. Electronic Journal of Contemporary Japanese Studies, Discussion Paper 5. http://www.japanesestudies.org.uk/discussionpapers/2006/Tran.html

[25] Heinze, U. and Thomas, P. (2014) Self and Salvation: Visions of Hikikomori in Japanese Manga. Journal of the German Institute for Japanese Studies Tokyo, 26, 151-169. http://www.degruyter.com/view/j/cj_cj.2014.26.issue-1_20140226101600/cj-2014-0007/cj-2014-0007.xml

[26] Chan, H. and Lo, T. (2014) Quality of Life of the Hidden Youth in Hong Kong. Applied Research in Quality of Life, 9, 951-969. http://link.springer.com/article/10.1007\%2Fs11482-013-9279-x\#/page-1 http://dx.doi.org/10.1007/s11482-013-9279-x

[27] Umeda, M. and Kawakami, N. (2012) 2002-2006 WMHJSG. Association of Childhood Family Environments with the Risk of Social Withdrawal ("Hikikomori") in the Community Population in Japan. Psychiatry and Clinical Neurosciences, 66, 121-129. http://dx.doi.org/10.1111/j.1440-1819.2011.02292.x http://onlinelibrary.wiley.com/doi/10.1111/j.1440-1819.2011.02292.x/epdf

[28] Kaneko, S. (2006) Japan’s “Socially Withdrawn Youths” and Time Constraints in Japanese Society-Management and Conceptualization of Time in a Support Group for "Hikikomori”. Time \& Society, 15, 233-249. http://dx.doi.org/10.1177/0961463X06067034

[29] Li, T.M. and Wong, P.W. (2015) Youth Social Withdrawal Behavior (Hikikomori): A Systematic Review of Qualitative and Quantitative Studies. Australia and New Zealand Journal of Psychiatry, 49, 595-609. http://dx.doi.org/10.1177/0004867415581179 http://anp.sagepub.com/cgi/pmidlookup?view=long\&pmid=25861794

[30] Teo, A.R. (2013) Social Isolation Associated with Depression: A Case Report of Hikikomori. International Journal of Social Psychiatry, 59, 339-341. http://isp.sagepub.com/cgi/pmidlookup?view=long\&pmid=22408115 http://dx.doi.org/10.1177/0020764012437128

[31] Teo, A.R., Fetters, M.D., Stufflebam, K., Tateno, M., Balhara, Y., Choi, T.Y., et al. (2015) Identification of the Hikikomori Syndrome of Social Withdrawal: Psychosocial Features and Treatment Preferences in Four Countries. International Journal of Social Psychiatry, 61, 64-72. http://isp.sagepub.com/cgi/pmidlookup?view=long\&pmid=24869848 http://dx.doi.org/10.1177/0020764014535758

[32] Nagata, T., Yamada, H., Teo, A.R., Yoshimura, C., Nakajima, T. and van Vliet, I. (2013) Comorbid Social Withdrawal (Hikikomori) in Outpatients with Social Anxiety Disorder: Clinical Characteristics and Treatment Response in a Case Series. International Journal of Social Psychiatry, 59, 73-78. http://dx.doi.org/10.1177/0020764011423184 http://isp.sagepub.com/cgi/pmidlookup?view=long\&pmid=21997765

[33] Lee, Y.S., Lee, J.Y., Choi, T.Y. and Choi, J.T. (2013) Home Visitation Program for Detecting, Evaluating and Treating Socially Withdrawn Youth in Korea. Psychiatry and Clinical Neurosciences, 67, 193-202. http://dx.doi.org/10.1111/pcn.12043

[34] Uchida, C. (2010) Apathetic and Withdrawing Students in Japanese Universities-With Regard to Hikikomori and Student Apathy. Journal of Medical and Dental Sciences, 57, 95-108. http://www.health.ibaraki.ac.jp/ibaraki_HP/2010.3JMDS.pdf

[35] Tateno, M., Park, T.W., Kato, T.A., Umene-Nakano, W. and Saito, T. (2012) Hikikomori as a Possible Clinical Term in Psychiatry: A Questionnaire Survey. BMC Psychiatry, 12, 169. http://dx.doi.org/10.1186/1471-244X-12-169 http://www.biomedcentral.com/1471-244X/12/169

[36] Charzman, K. (2008) Constructionism and the Grounded Theory Method. In: Holstein, J.A. and Gubrium, J.F., Eds., Handbook of Constructionist Research, Guilford Press, New York, 397-412. http://www.sxf.uevora.pt/wp-content/uploads/2013/03/Charmaz_2008-a.pdf 
[37] Tong, A., Sainsbury, P. and Craig, J. (2007) Consolidated Criteria for Reporting Qualitative Research (COREQ): A 32Item Checklist for Interviews and Focus Groups. International Journal for Quality in Health Care, 19, 349-357. http://intqhc.oxfordjournals.org/content/19/6/349.full-text.pdf http://dx.doi.org/10.1093/intqhc/mzm042

[38] Genda, Y. (2007) Jobless Youths and the NEET Problem in Japan. Social Science Japan Journal, 10, 23-40. http://ssij.oxfordjournals.org/content/10/1/23.full.pdf+html http://dx.doi.org/10.1093/ssji/jym029 
Appendix. Non-sampling data collection and review.

News

Related articles

Electronic dictionary

Documentary

Fictional

Non-fictional

Creative production
1. Anonymous. Nearly one in ten teenagers have dropped out as "Neets"; Politics. Birmingham Post June 20, 2008, 2008.

2. Chan J. Random Killing in Tokyo points to deepening social crisis in Japan. World Socialist Web Site June 23, 2008, 2008.

3. Dyer G. Japan—land of setting sun; Global recession could hasten twilight for Asian political powerhouse; Endangered species. Taranaki Daily News 2009: 15.

4. Editors. 2004; http://www.humiliationstudies.org/news-old/archives/2004_06.html.

5. Flockhart G. More for your Pound at Lyceum. Evening News 2007: 6

6. Goldberg J. Answering Machine: Dice-K Comes Prepared. Knight Ridder Tribune Business News 2007: 1.

7. Howe K. Millions In Japan Living In Shadow: Former Toyko

journalist describes dysfunction following economic slump. Knight Ridder Tribune Business News 2007:1.

8. James V. Japan's youth runs out of control. New Statesman 2001; 14(673): 23.

9. Lewin T. At Colleges, Women Are Leaving Men in the Dust. New York Times 2006: 1.1.

10. Zielenziger M. Reclusive Young Adults Reflect Anxious Japanese Nation. Knight Ridder Tribune Business News 2002: 1 .

11. Nakamoto M. Eli Lilly looks to lift Japan with Prozac. Financial Times 2001: 04.

12. News of Hikikomori [Hikikomori] Jouhou: The Voice of Your Heart.

http://www.nhk.or.jp/fnet/hikikomori/2003/column/index.html. Accessed 10 July, 2009.

13. Penn W. Televiews/Thirsty for fame: Young announcer deserves a drink. The Daily Yomiuri Nov 1, 2001. 2001: 1 .

14. Rees P. Japan: The Missing Million. BBC News World Edition 20 Oct 2002, 2002.

15. Secher B. Out of sight, not out of mind. Asahi Evening News 2002: 1.

16. Otake T. Japan Times, Tokyo, WEEK 3 column:bizarre bouts of self-expression. Knight Ridder Tribune Business News 2006: 1.

17. Larimer T. Natural-born killers? Time 2000;156(9): 37.

18. Jones M. For some in Japan, a room is their world. International Herald Tribune Jan 14, 2006, $2006: 1$.

19. Jones M. Shutting Themselves In. New York Times Magazine 2006: 46.

20. Vickery D. Hikikomori. 2006; http://vickery.dk/hikikomori/.

21. Colin J. Seven die in group suicide: Young Japanese are believed to have met on Internet. National Post 2004: A.16.

22. Lo A. Living in Tokyo’s shadow. South China Morning Post 2005: 14.

23. Moy P. Agency fears 6000 young hiding from society; Study of secondary schools finds some troubled youth acting like hermits. South China Morning Post July 18, 2005, 2005: 1.

24. Ng KC. More youngsters hide out at home Survey shows threefold rise in urban hermits. South China Morning Post Jan 15, 2007, 2007: 3.

25. Ryall J. Help is on the way for Japanese who shut their door to the world. South China Morning Post Apr 21, 2003, 2003: 7.

26. Zhuang P. Study identifies teenagers at risk of becoming hermits. South China Morning Post Sep 11, 2006, 2006: 1.

27. Anonymous. Out of sight, not out of mind. Asahi Evening News 2002: 1.

28. Anonymous. There's no place like home. Asahi Evening News 2002: 1.

29. Anonymous. Web site set up to support socially isolated people. Mainichi Daily News 2004: 1.

30. Anonymous. Coffee shop opens doors to hermits in Kyoto. Mainichi Daily News 2006: 1.

31. Bloomberg News. Asia; Japan: Consumer Confidence Falls. New York Times 2008.

32. Walsh J. Shyness and social phobia-A social work perspective on a problem in living. Health \& Social Work 2002; 27(2): 137-144.

33. Colman AM. In: Colman AM, ed. A Dictionary of Psychology in Politics \& Social Sciences 3rd ed. Oxford (UK): Oxford University Press; 2009.

34. Karman K. Hikikomori [Documentary] 2004.

35. Murakami R. Symbiosis Worm. Taipei Shi: Da tian chu ban you xian gong si,; 2002.

36. Zielenziger M. Shutting out the sun: How Japan created its own lost generation. 1st ed. New York: Nan A. Talese; 2006.

37. Yamamoto Y. NHK Yokoso-Welcome to NHK (Nippon Hikikomori Kyokai). July 9, 2006, 2006. 38. Nakajima R. Ore tachi no sekai/This World of Ours 2008. 